\title{
BOUNDARY OPERATORS IN EUCLIDEAN QUANTUM GRAVITY
}

\author{
Ivan G Avramidi *, Giampiero Esposito and Alexander Yu Kamenshchik \\ Department of Mathematics, University of Greifswald, Jahnstr. 15a, 17487 Greifswald, \\ Germany \\ Istituto Nazionale di Fisica Nucleare, Sezione di Napoli, Mostra d'Oltremare Padiglione \\ 20, 80125 Napoli, Italy \\ Dipartimento di Scienze Fisiche, Mostra d'Oltremare Padiglione 19, 80125 Napoli, Italy \\ Nuclear Safety Institute, Russian Academy of Sciences, 52 Bolshaya Tulskaya, Moscow \\ 113191, Russia
}

\begin{abstract}
Gauge-invariant boundary conditions in Euclidean quantum gravity can be obtained by setting to zero at the boundary the spatial components of metric perturbations, and a suitable class of gauge-averaging functionals. This paper shows that, on choosing the de Donder functional, the resulting boundary operator involves projection operators jointly with a nilpotent operator. Moreover, the elliptic operator acting on metric perturbations is symmetric. Other choices of mixed boundary conditions, for which the normal components of metric perturbations can be set to zero at the boundary, are then analyzed in detail.

* Alexander von Humboldt Fellow. On leave of absence from Research Institute for Physics, Rostov State University, Stachki 194, 344104 Rostov-on-Don, Russia. E-mail: avramidi@math-inf.uni-greifswald.d400.de
\end{abstract}




\section{Boundary operators in Euclidean quantum gravity}

Last, the evaluation of the 1-loop divergence in the axial gauge for gravity is obtained. Interestingly, such a divergence turns out to coincide with the one resulting from transversetraceless perturbations.

PACS numbers: 0370, 0460

\section{Introduction}

Over the last few years, a substantial progress has been made in the understanding of the asymptotic heat kernel with pure and mixed boundary conditions in quantum field theory. In particular, whenever the boundary conditions for spinor fields, gauge fields and gravitation are expressed in terms of complementary projection operators [1], the geometric form of the 1-loop divergences is by now well understood [2-4], and it agrees with the results obtained by analytic techniques [5-8]. What happens is that the volume part of such 1-loop divergences involves the curvature of the background, whilst the surface part involves both the extrinsic and the intrinsic curvature tensor of the boundary and the projection operators occurring in the boundary conditions [2-4].

In Euclidean quantum gravity, however, a more general scheme can be considered. As it has been shown in $[8,9]$, which rely on the work in $[10]$, one can set to zero at the boundary $\partial M$ the spatial components $h_{i j}$ of the metric perturbations $h_{a b}$, jointly with any gauge-averaging functional $\Phi_{a}(h)$ which leads to a well defined spectrum of eigenvalues (hereafter, lower-case indices $a, b$ should be regarded as abstract indices for fourdimensional tensor fields). On requiring the invariance of such boundary conditions under 


\section{Boundary operators in Euclidean quantum gravity}

local transformations of metric perturbations, i.e. $\delta h_{a b}=\nabla_{(a} \varphi_{b)}$, one finds that a necessary and sufficient condition for this is that the whole ghost 1-form should vanish at the boundary $[8,9]$. In particular, the background 4-manifold $M$ can be taken to be flat Euclidean 4-space bounded by a 3-sphere. The analysis of flat backgrounds is indeed relevant both for Euclidean field theory [11] and for the analysis of massless supergravity models in the presence of boundaries [12]. In the de Donder gauge, the boundary conditions on the metric perturbations which are invariant under gauge transformations take then the form $[8,9]$

$$
\begin{gathered}
{\left[h_{i j}\right]_{\partial M}=0} \\
{\left[\frac{\partial h_{00}}{\partial \tau}+\frac{6}{\tau} h_{00}-\frac{\partial}{\partial \tau}\left(g^{i j} h_{i j}\right)+\frac{2}{\tau^{2}} h_{0 i} \mid i\right]_{\partial M}=0} \\
{\left[\frac{\partial h_{0 i}}{\partial \tau}+\frac{3}{\tau} h_{0 i}-\frac{1}{2} h_{00 \mid i}\right]_{\partial M}=0}
\end{gathered}
$$

where $i, j=1,2,3$, and $g^{i j}$ are the spatial components of the contravariant form of the background 4-metric. They are used to raise indices of $h_{i j}$, whilst the covariant $g_{i j}$ lowers indices of $h^{i j}$. Moreover, with a standard notation, $\tau=x^{0}$ is the radial coordinate, $\hat{x}^{i}$ are local coordinates on the 3 -sphere boundary with unit metric $c_{i j}(\hat{x})$, so that locally

$$
g=d \tau \otimes d \tau+\tau^{2} c_{i j}(\hat{x}) d \hat{x}^{i} \otimes d \hat{x}^{j}
$$

and the stroke denotes covariant differentiation tangentially with respect to the Levi-Civita connection of the boundary. As we said before, the whole ghost 1-form should vanish at the boundary. 


\section{Boundary operators in Euclidean quantum gravity}

Although the corresponding 1-loop divergence was already evaluated in [8] by means of the regularization algorithm introduced in [13], the geometric counterpart of such an analytic investigation remains unknown in the literature (cf [14]). Note that (1.2) and (1.3) involve both normal and tangential derivatives of $h_{00}$ and $h_{0 i}$, and are not expressed in terms of (complementary) projection operators.

Thus, to complete the analysis of gauge-invariant boundary conditions in Euclidean quantum gravity, it appears crucial to perform a geometric analysis of the quantum boundary-value problem corresponding to (1.1)-(1.3). For this purpose, section 2 describes the general framework for gauge-invariant boundary conditions in Euclidean quantum gravity. Section 3 studies the projection and nilpotent operators occurring in the de Donder case. Section 4 obtains an equivalent form of the boundary conditions of section 3 , which makes it easier to compare them with other sets of mixed boundary conditions studied in the literature. Section 5 proves symmetry of the Laplace operator when the boundary conditions (1.1)-(1.3) are imposed. Section 6 is instead devoted to the analysis of boundary operators when the normal components of metric perturbations are set to zero at the boundary. Section 7 evaluates the 1-loop divergence for pure gravity in the axial gauge. Concluding remarks are presented in section 8.

\section{Gauge-invariant boundary conditions for Euclidean quantum gravity}

For gauge fields and gravitation the boundary conditions should be gauge-invariant under local gauge transformations with some suitable boundary conditions on the corresponding gauge functions (ghost fields). This is why the boundary conditions should be mixed, in that some components of the field obey a set of boundary conditions (say, Dirichlet), and 


\section{Boundary operators in Euclidean quantum gravity}

the remaining part of the field obeys another set of boundary conditions (Neumann or Robin).

We are here interested in the derivation of mixed boundary conditions for Euclidean quantum gravity. The Euclidean formulation is essential to obtain well posed boundaryvalue problems for elliptic operators. Its relevance for the Lorentzian theory deserves further investigation [8], since no general result holds which relates Lorentzian and Riemannian curved four-manifolds through a Wick rotation, and the corresponding Green functions [11]. One can say, however, that our investigation of flat backgrounds can be applied to put on solid ground the analysis of ultraviolet divergences in quantum field theory on manifolds with boundary.

The knowledge of the classical variational problem, and the principle of gauge invariance, are enough to lead to a highly non-trivial quantum boundary-value problem. Indeed, it is by now well known that, if one fixes the 3 -metric at the boundary in general relativity, the corresponding variational problem is well posed and leads to the Einstein equations, providing the Einstein-Hilbert action is supplemented by a boundary term whose integrand is proportional to the trace of the second fundamental form [15]. In the corresponding quantum boundary-value problem, which is relevant for the 1-loop approximation in quantum gravity, the perturbations $h_{i j}$ of the induced 3-metric are set to zero at the boundary. Moreover, the whole set of metric perturbations $h_{a b}$ are subject to the infinitesimal gauge transformations

$$
{ }^{\varphi} h_{a b} \equiv h_{a b}+\delta h_{a b}=h_{a b}+\nabla_{(a} \varphi_{b)}
$$




\section{Boundary operators in Euclidean quantum gravity}

where $\nabla$ is the Levi-Civita connection of the background 4-geometry with metric $g$, and $\varphi_{a} d x^{a}$ is the ghost 1 -form. In geometric language, the infinitesimal difference between ${ }^{\varphi} h_{a b}$ and $h_{a b}$ is given by the Lie derivative along $\varphi$ of the 4-metric $g$.

For problems with boundaries, equation (2.1) implies that

$$
{ }^{\varphi} h_{i j}=h_{i j}+\varphi_{(i \mid j)}+K_{i j} \varphi_{0}
$$

where $K_{i j}$ is the extrinsic-curvature tensor of the boundary. Of course, $\varphi_{0}$ and $\varphi_{i}$ are the normal and tangential components of the ghost 1-form, respectively. Note that boundaries make it necessary to perform a $3+1$ split of space-time geometry and physical fields. As such, they introduce non-covariant elements in the analysis of problems relevant for quantum gravity. This seems to be an unavoidable feature, although the boundary conditions may be written in a covariant way (see sections 3 and 4).

In the light of (2.2), the boundary conditions

$$
\left[h_{i j}\right]_{\partial M}=0
$$

are gauge-invariant, i.e.

$$
\left[{ }^{\varphi} h_{i j}\right]_{\partial M}=0
$$

if and only if the whole ghost 1-form obeys homogeneous Dirichlet conditions, so that

$$
\begin{gathered}
{\left[\varphi_{0}\right]_{\partial M}=0} \\
{\left[\varphi_{i}\right]_{\partial M}=0 .}
\end{gathered}
$$




\section{Boundary operators in Euclidean quantum gravity}

The conditions (2.4) and (2.5) are necessary and sufficient since $\varphi_{0}$ and $\varphi_{i}$ are independent, and three-dimensional covariant differentiation commutes with the operation of restriction at the boundary. Indeed, we are assuming that the boundary is smooth and not totally geodesic, i.e. $K_{j l} \neq 0$. However, at those points of $\partial M$ where the extrinsic-curvature tensor vanishes, the condition (2.4) is no longer necessary.

The problem now arises to impose boundary conditions on the remaining set of metric perturbations. The key point is to make sure that the invariance of such boundary conditions under the transformations (2.1) is again guaranteed by (2.4) and (2.5), since otherwise one would obtain incompatible sets of boundary conditions on the ghost 1-form. Indeed, on using the Faddeev-Popov formalism for the amplitudes of quantum gravity, it is necessary to use a gauge-averaging term in the Euclidean action, of the form

$$
I_{\text {g.a. }} \equiv \frac{1}{32 \pi G \alpha} \int_{M} \Phi_{a}(h) \Phi^{a}(h) \sqrt{g} d^{4} x
$$

where $G$ is Newton's constant, $\Phi_{a}(h)$ is any gauge-averaging functional which leads to selfadjoint elliptic (and hence non-degenerate) operators on metric and ghost perturbations, and $\alpha$ is an arbitrary dimensionless parameter. As in all our analysis, $\sqrt{g} d^{4} x$ is the invariant integration measure with respect to the background 4-metric. In particular, if the de Donder gauge is chosen, i.e. (with $a, b=0,1,2,3$ )

$$
\Phi_{a}^{d D}(h) \equiv E_{a}{ }^{b c f} \nabla_{b} h_{c f}=\nabla^{b}\left(h_{a b}-\frac{1}{2} g_{a b} g^{c f} h_{c f}\right)
$$

where $E^{a b c d} \equiv g^{a(c} g^{d) b}-\frac{1}{2} g^{a b} g^{c d}$, one finds that

$$
\delta \Phi_{a}^{d D} \equiv \Phi_{a}^{d D}(h)-\Phi_{a}^{d D}\left({ }^{\varphi} h\right)=-\frac{1}{2}\left(g_{a}{ }^{b} \square+R_{a}{ }^{b}\right) \varphi_{b}
$$


Boundary operators in Euclidean quantum gravity

where $\square \equiv g^{a b} \nabla_{a} \nabla_{b}$, and $R_{a b}$ is the Ricci tensor of the background. The operator $-\left(g_{a}{ }^{b} \square+R_{a}{ }^{b}\right)$ is elliptic and, of course, acts linearly on the ghost 1 -form. Thus, if one imposes the boundary conditions

$$
\begin{gathered}
{\left[\Phi_{0}^{d D}(h)\right]_{\partial M}=0} \\
{\left[\Phi_{i}^{d D}(h)\right]_{\partial M}=0}
\end{gathered}
$$

their invariance under (2.1) is guaranteed when (2.4) and (2.5) hold, by virtue of (2.8). Hence one also has

$$
\begin{gathered}
{\left[\Phi_{0}^{d D}\left({ }^{\varphi} h\right)\right]_{\partial M}=0} \\
{\left[\Phi_{i}^{d D}\left({ }^{\varphi} h\right)\right]_{\partial M}=0 .}
\end{gathered}
$$

Note that the boundary conditions on the ghost 1-form become redundant if one also imposes the conditions $(2.3 \mathrm{~b}),(2.9 \mathrm{~b})$ and $(2.10 \mathrm{~b})$. Nevertheless, we shall always write them explicitly, since the ghost 1-form plays a key role in quantum gravity.

Of course, the most general scheme does not depend on the choice of the de Donder term (see section 5$)$, so that it relies on $(2.3 \mathrm{a}),(2.3 \mathrm{~b}),(2.4),(2.5)$, jointly with

$$
\begin{gathered}
{\left[\Phi_{0}(h)\right]_{\partial M}=0} \\
{\left[\Phi_{0}\left({ }^{\varphi} h\right)\right]_{\partial M}=0} \\
{\left[\Phi_{i}(h)\right]_{\partial M}=0}
\end{gathered}
$$


Boundary operators in Euclidean quantum gravity

$$
\left[\Phi_{i}\left({ }^{\varphi} h\right)\right]_{\partial M}=0
$$

Again, it is enough to write (2.3a), (2.11a), (2.12a), (2.4), (2.5), or (2.3a), (2.3b) jointly with (2.11a), (2.11b) and (2.12a), (2.12b).

\section{Projection and nilpotent operators}

Following [8, 9], we study the Barvinsky boundary conditions of section 2 for the semiclassical 〈out|in〉 amplitude of Euclidean quantum gravity when a flat four-dimensional background $(M, g)$ is bounded by a smooth three-dimensional boundary $(\partial M, \gamma)$. The analysis in arbitrary $d$-dimensional flat manifolds with smooth $(d-1)$-dimensional boundary can be performed along the same lines.

As the first step in our geometric analysis, we have to re-express such boundary conditions in a manifestly covariant way. For this purpose, we consider the four-dimensional tensor field $q$ on $(M, g)$ defined as

$$
q_{a b} \equiv g_{a b}-n_{a} n_{b}
$$

whose restriction to $(\partial M, \gamma)$ coincides with the metric $\gamma_{i j}$ on $\partial M$. Here, $n^{a}$ is the inward pointing normal to $\partial M$ with unit norm, i.e. $n_{a} n^{a}=1$. Of course, $q_{a b}$ is a projector of vector fields onto the surface $\Sigma$ orthogonal to the normal vector $n^{a}$, i.e. $q_{a b} n^{b}=0$. The boundary conditions $(2.3 \mathrm{a})$ are then expressed as

$$
[\Pi h]_{\partial M}=0
$$

where $\Pi$ is a projector of symmetric 2 -forms onto $\partial M$, defined as

$$
\Pi_{a b}^{c d} \equiv q_{(a}^{c} q_{b)}^{d}
$$




\section{Boundary operators in Euclidean quantum gravity}

In the following we choose the de Donder gauge-averaging functional defined in (2.7). Given the Levi-Civita connection $\nabla$ of the background, the introduction of the differential operators $\nabla_{(n)}$ and $\widetilde{\nabla}_{a}$ defined as

$$
\begin{aligned}
& \nabla_{(n)} \equiv n^{a} \nabla_{a} \\
& \widetilde{\nabla}_{a} \equiv q_{a}^{b} \nabla_{b}
\end{aligned}
$$

makes it now possible to write the covariant form of (2.9a) and (2.10a) as

$$
\left[\left(A \nabla_{(n)}+B^{e} \widetilde{\nabla}_{e}\right) h\right]_{\partial M}=0
$$

where the matrices $A$ and $B^{e}$ turn out to be

$$
\begin{gathered}
A_{a b}^{c d} \equiv n_{a} n_{b}\left(n^{c} n^{d}-q^{c d}\right)+2 n_{(a} q_{b)}^{(c} n^{d)} \\
B_{a b}{ }^{c d, e} \equiv 2 n_{a} n_{b} n^{(c} q^{d) e}-n_{(a} q_{b)}^{e} n^{c} n^{d} \\
+2 n_{(a} q_{b)}^{(c} q^{d) e}-n_{(a} q_{b)}^{e} q^{c d} .
\end{gathered}
$$

Interestingly, a peculiar property of this set of boundary conditions is that $A$ and $B^{e}$ are not symmetric under the interchange of $a b$ and $c d$, and $A$ is not a projection operator. By contrast, $\Pi$ is symmetric under the above interchange, and is a projector by definition.

One should also bear in mind that, for any $d$-dimensional background $(d=4$ in our case), the following property holds:

$$
\operatorname{rank}(A)+\operatorname{rank}(\Pi)=\frac{d(d+1)}{2}
$$




\section{Boundary operators in Euclidean quantum gravity}

This condition ensures that the gauge-invariant boundary conditions (3.2) and (3.6) are complete in that they fix all components of metric perturbations, and do not introduce any spurious restrictions which would lead to an overdetermined problem.

Note that one can decompose the matrix $A$ in the form

$$
A=\pi+p-\nu
$$

where the matrices $\pi, p$ and $\nu$ are defined by

$$
\begin{gathered}
\pi_{a b}^{c d} \equiv n_{a} n_{b} n^{c} n^{d} \\
p_{a b}^{c d} \equiv 2 n_{(a} q_{b)}^{(c} n^{d)} \\
\nu_{a b}^{c d} \equiv n_{a} n_{b} q^{c d} .
\end{gathered}
$$

It is easy to see that $\Pi, \pi$ and $p$ are projection operators, i.e.

$$
\begin{gathered}
\Pi^{2}=\Pi \quad \pi^{2}=\pi \quad p^{2}=p \\
\Pi \pi=\pi \Pi=\Pi p=p \Pi=\pi p=p \pi=0 \\
\Pi+\pi+p=\mathbb{I}
\end{gathered}
$$

$\mathbb{I}$ being the identity matrix in the vector space of symmetric 2 -forms, $\mathbb{I}_{a b}{ }^{c d} \equiv \delta_{(a}^{c} \delta_{b)}^{d}$, whereas the matrix $\nu$ is not a projector but a nilpotent matrix, i.e.

$$
\nu^{2}=0
$$

which is orthogonal to $p$

$$
p \nu=\nu p=0
$$


whilst

$$
\begin{aligned}
& \pi \nu=\nu \\
& \nu \pi=0 .
\end{aligned}
$$

Moreover, the projector $\Pi$ annihilates $\nu$ from the left, $\Pi \nu=0$, but not in the reverse order, since $\nu \Pi=\nu$. By virtue of (3.17)-(3.20), one has

$$
A \nu=\nu
$$

whilst

$$
\nu A=0
$$

In the light of (3.10), (3.16) and (3.17) one sees immediately that the matrix

$$
\Pi+A=\mathbb{I}-\nu
$$

is not degenerate and has the inverse

$$
(\Pi+A)^{-1}=\mathbb{I}+\nu
$$

Thus, the action of $A$ and $B^{e}$ on $h$ yields tensor fields which are orthogonal to $\Pi$, i.e.

$$
\begin{gathered}
\Pi A=0 \\
\Pi B^{e}=0 .
\end{gathered}
$$

On the other hand, $A$ and $B^{e}$ do not commute with $\Pi$, and hence one finds that

$$
A \Pi=-\nu
$$


Boundary operators in Euclidean quantum gravity

$$
B_{a b}^{c d, e} \Pi_{c d}^{f g}=2 n_{(a} q_{b)}^{(f} q^{g) e}-n_{(a} q_{b)}^{e} q^{f g} .
$$

By virtue of (3.25) and (3.26), it is possible to express $A$ and $B^{e}$ as

$$
\begin{aligned}
A & =(\mathbb{I}-\Pi) A \\
B^{e} & =(\mathbb{I}-\Pi) B^{e} .
\end{aligned}
$$

Thus, an equivalent expression of the boundary conditions (3.6) is

$$
\left[(\mathbb{I}-\Pi)\left(A \nabla_{(n)}+B^{e} \widetilde{\nabla}_{e}\right) h\right]_{\partial M}=0 .
$$

\section{Equivalent form of the boundary conditions}

It is now convenient to transform slightly the form of the boundary conditions. This makes it easier to compare our analysis with previous work in the literature [14], and can be applied to the geometric analysis of heat-kernel asymptotics (cf [2-4]). For this purpose, let us define the matrix $E=\left(E_{a b}{ }^{c d}\right)$ with elements

$$
E_{a b}^{c d} \equiv \delta_{(a}^{c} \delta_{b)}^{d}-\frac{1}{2} g_{a b} g^{c d} .
$$

Substituting here $g_{a b}=q_{a b}+n_{a} n_{b}$ we obtain the matrix $E$ in the form

$$
E=\mathbb{I}-\frac{1}{2}\left(\nu+\nu^{T}\right)-\frac{1}{2} \pi-\frac{1}{2} V .
$$

where $T$ denotes the transposition, and the matrix $V$ is defined by

$$
V_{a b}^{c d} \equiv q_{a b} q^{c d} .
$$




\section{Boundary operators in Euclidean quantum gravity}

Now, using the formulae of the previous section, we obtain easily

$$
(\mathbb{I}-\Pi) E=p+\frac{1}{2}(\pi-\nu) .
$$

It is not difficult to see that this can be expressed in terms of the matrix $A$

$$
(\mathbb{I}-\Pi) E=\frac{1}{2}(\mathbb{I}+p) A .
$$

Noting that

$$
(\mathbb{I}+p)^{-1}=\mathbb{I}-\frac{1}{2} p
$$

we find from (4.5)

$$
A=2\left(\mathbb{I}-\frac{1}{2} p\right)(\mathbb{I}-\Pi) E .
$$

Therefore, the boundary conditions (3.6) (or (3.31)) can be re-written in the form

$$
\left[(\mathbb{I}-\Pi)\left(E \nabla_{(n)}+\frac{1}{2}(\mathbb{I}+p) B^{e} \widetilde{\nabla}_{e}\right) h\right]_{\partial M}=0 .
$$

Further we transform the operator $\widetilde{\nabla}_{e}$ as follows:

$$
\widetilde{\nabla}_{e}=\widetilde{\nabla}_{e}(\mathbb{I}-\Pi)+\widetilde{\nabla}_{e} \Pi=\left((\mathbb{I}-\Pi) \widetilde{\nabla}_{e}-\left(\widetilde{\nabla}_{e} \Pi\right)\right)(\mathbb{I}-\Pi)+\widetilde{\nabla}_{e} \Pi
$$

Taking into account the boundary condition (3.2) on the spatial components of $h$, one finds

$$
\left[\widetilde{\nabla}_{e} h\right]_{\partial M}=\left[\left((\mathbb{I}-\Pi) \widetilde{\nabla}_{e}-\left(\widetilde{\nabla}_{e} \Pi\right)\right)(\mathbb{I}-\Pi) h\right]_{\partial M} .
$$


Thus, the boundary conditions take the form

$$
\begin{gathered}
{[\Pi h]_{\partial M}=0} \\
{\left[(\mathbb{I}-\Pi)\left(E \nabla_{(n)}+F^{e} \widetilde{\nabla}_{e}+\widetilde{\nabla}_{e} F^{e}+D\right) h\right]_{\partial M}=0}
\end{gathered}
$$

where

$$
\begin{gathered}
F^{e} \equiv \frac{1}{4}(\mathbb{I}+p) B^{e}(\mathbb{I}-\Pi) \\
D \equiv-\frac{1}{2}(\mathbb{I}+p) B^{e}\left(\widetilde{\nabla}_{e} \Pi\right)(\mathbb{I}-\Pi)-(\mathbb{I}-\Pi)\left(\widetilde{\nabla}_{e} F^{e}\right)(\mathbb{I}-\Pi) .
\end{gathered}
$$

Using the explicit formulae of the previous section for the matrices $B^{e}$ and $\Pi$ one obtains

$$
\begin{gathered}
F_{a b}{ }^{c d, e}=\frac{1}{2} n_{a} n_{b} n^{(c} q^{d) e}-\frac{1}{2} n_{(a} q_{b)}^{e} n^{c} n^{d} \\
D_{a b}{ }^{c d}=2 n_{(a} q_{b)}^{(c} n^{d)} \operatorname{Tr} K .
\end{gathered}
$$

It is easy to see that the matrix $D$ is proportional to the projector $p$

$$
D=p \operatorname{Tr} K
$$

These boundary conditions are similar to the mixed form of generalized boundary conditions considered in [14]. The geometric theory of heat-kernel asymptotics resulting from (4.11) and (4.12) remains unknown, and is a difficult task in Euclidean quantum gravity.

Note that the matrix $F^{e}$ is antisymmetric and the matrix $D$ is symmetric with respect to the interchange of the pairs of indices $a b$ and $c d$, i.e.

$$
F^{a b c d, e}=-F^{c d a b, e}
$$


Boundary operators in Euclidean quantum gravity

$$
D^{a b c d}=D^{c d a b}
$$

and that they are both orthogonal to the projector $\Pi$

$$
\begin{gathered}
F^{e} \Pi=\Pi F^{e}=0 \\
D \Pi=\Pi D=0 .
\end{gathered}
$$

\section{Symmetry of the Laplace operator}

A crucial point in our analysis is the proof that the boundary conditions (4.11), (4.12) lead to a self-adjoint operator on metric perturbations. The de Donder gauge-averaging term

has the effect of reducing such an operator to the Laplace operator $-\square \equiv-g^{a b} \nabla_{a} \nabla_{b}$, where $\nabla_{a}$ denotes covariant differentiation with respect to the Levi-Civita connection of the background $M$. As a first step, one has to prove that $\square$ is symmetric. This means that, denoting by $\eta$ and $h$ any two elements of the space $\mathcal{D}(M)$ of $C^{\infty}$, symmetric tensor fields on $(M, g)$ of type $(0,2)$, and defining (see (4.1))

$$
(\eta, h) \equiv \int_{M} d^{4} x \sqrt{g}<\eta, E h>
$$

where

$$
<\eta, E h>\equiv \eta_{a b} E^{a b c d} h_{c d}
$$

the following property should hold:

$$
I(\eta, h) \equiv(\eta, \square h)-(\square \eta, h)=0
$$

for all $\eta, h \in \mathcal{D}(M)$ and obeying the boundary conditions (4.11), (4.12). 
In general, the left-hand side of (5.3) takes the form

$$
I(\eta, h)=\int_{\partial M}\left[<\eta, E \nabla_{(n)} h>-<\nabla_{(n)} \eta, E h>\right] \sqrt{\gamma} d^{3} x
$$

where $\gamma$ is the determinant of the 3-metric of the boundary. In our boundary-value problem, spatial perturbations $\Pi \eta$ and $\Pi h$ are set to zero at the boundary (see (4.11)), and hence only the normal components $(\mathbb{I}-\Pi) \eta$ and $(\mathbb{I}-\Pi) h$ contribute to (5.3). Therefore, one has

$$
\begin{aligned}
I(\eta, h) & =\int_{\partial M}\left[<\eta,(\mathbb{I}-\Pi) E \nabla_{(n)} h>\right. \\
& \left.-<(\mathbb{I}-\Pi) E \nabla_{(n)} \eta, h>\right] \sqrt{\gamma} d^{3} x .
\end{aligned}
$$

Using now the second boundary condition (4.12) one obtains

$$
I(\eta, h)=\int_{\partial M}[<\eta, \Lambda h>-<\Lambda \eta, h>] \sqrt{\gamma} d^{3} x
$$

where

$$
\Lambda \equiv(\mathbb{I}-\Pi)\left(F^{e} \widetilde{\nabla}_{e}+\widetilde{\nabla}_{e} F^{e}+D\right)(\mathbb{I}-\Pi)
$$

is a first-order differential operator on the boundary. Integrating by parts it is immediately seen that this operator is symmetric

$$
\Lambda^{\dagger}=\Lambda
$$

by virtue of the antisymmetry of the matrix $F^{e}$ and the symmetry of the matrix $D$. Thus, the antisymmetric form $I(\eta, h)$ vanishes, and this proves that the Laplacian with the boundary conditions (4.11), (4.12) is symmetric. In a non-covariant analysis, the 
imposition of the boundary conditions in the form $(1.1)-(1.3)$ shows that $I(\eta, h)$ reduces

to the integral over $\partial M$ of the total divergence $\left[-\eta_{00} h^{0 i}+h_{00} \eta^{0 i}\right]_{\mid i}$, and hence vanishes by virtue of Stokes' theorem (and bearing in mind that $\partial \partial M=0$ ).

The task now remains to prove that self-adjoint extensions exist and are unique. This appears feasible, since one deals with a Laplace operator with a Dirichlet sector resulting from (4.11). Nevertheless, (5.6) already expresses a non-trivial property: mixed boundary conditions which are completely invariant under infinitesimal diffeomorphisms can be consistently imposed in Euclidean quantum gravity.

\section{Other choices of mixed boundary conditions}

The technical problems of section 4 in obtaining the geometric form of heat-kernel asymptotics result from an involved set of mixed boundary conditions on the normal components of metric perturbations. Hence we now study boundary operators whose action on $h_{00}$ and $h_{0 i}$ is instead very simple. The first set of boundary conditions is the covariant version of those analyzed in [16]. They read

$$
\begin{gathered}
{\left[n^{b} h_{a b}\right]_{\partial M}=0} \\
{\left[\left(\nabla_{(n)}+\frac{(2+u)}{3}(\operatorname{Tr} K)\right)\left(\Pi_{a b}^{c d} h_{c d}\right)\right]_{\partial M}=0}
\end{gathered}
$$

where $u$ is a dimensionless parameter. The non-covariant formulation of (6.2) requires that $\frac{\partial h_{i j}}{\partial \tau}+\frac{u}{\tau} h_{i j}$ should vanish at the boundary. Hence one is dealing with Robin conditions on $h_{i j}[16]$. Note that this is not the Barvinsky framework. We are still using the de 


\section{Boundary operators in Euclidean quantum gravity}

Donder gauge-averaging functional, and hence the operator acting on metric perturbations reduces to $-\square$ in our flat Euclidean background. The boundary conditions (6.1) and (6.2) represent the extension to gravity of the scheme used in setting electric boundary conditions for Euclidean Maxwell theory. However, unlike Maxwell's theory, they are not completely gauge-invariant [16]. When $u=0,(6.2)$ sets to zero at the boundary the linearized magnetic curvature, obtained out of the Weyl tensor [17]. Moreover, the lack of complete gauge invariance of the boundary conditions implies that, even on the mass shell, transition amplitudes may depend on the specific form of the gauge-averaging functional.

According to the definition (5.1), one thus finds that the operator $-\square$ is symmetric if and only if the following surface integral vanishes:

$$
\begin{aligned}
I_{B} & \equiv(\eta, \square h)-(\square \eta, h) \\
& =\int_{\partial M}\left[\eta^{i j} \nabla_{(n)}\left(h_{i j}-\frac{1}{2} g_{i j} \hat{h}\right)-h^{i j} \nabla_{(n)}\left(\eta_{i j}-\frac{1}{2} g_{i j} \hat{\eta}\right)\right] \sqrt{\gamma} d^{3} x
\end{aligned}
$$

where $\hat{h} \equiv g^{a b} h_{a b}, \hat{\eta} \equiv g^{a b} \eta_{a b}$. In fact, it is obvious that the boundary conditions (6.1), (6.2) do satisfy this condition and hence lead to a symmetric Laplace operator, since the integrand in (6.3) is a linear combination of $\eta^{i j} h_{i j}$ and $\hat{\eta} \hat{h}$ with vanishing coefficients.

In the Barvinsky framework, the boundary conditions (6.1) may still be obtained if one uses the axial gauge-averaging functional $\Phi_{a}^{A}(h) \equiv n^{b} h_{a b}$. The resulting ghost operator takes the form

$$
\mathcal{F}_{a}^{b}=\left(\delta_{a}^{b}+n_{a} n^{b}\right) \nabla_{(n)}+n^{b} \widetilde{\nabla}_{a}
$$

with Dirichlet boundary conditions (2.4) and (2.5) on the ghost field. It is not difficult to show that with Dirichlet boundary conditions the ghost operator (6.4) does not have any 


\section{Boundary operators in Euclidean quantum gravity}

eigenfunctions at all. Indeed, consider the eigenvalue equation

$$
\mathcal{F} \varphi_{\lambda}=\lambda \varphi_{\lambda} .
$$

The solution of this equation in the coordinates $\tau, \hat{x}$ takes the form

$$
\begin{gathered}
\varphi_{0_{\lambda}}(\tau, \hat{x})=\exp \left(\frac{1}{2} \lambda \tau\right) f_{0_{\lambda}}(\hat{x}) \\
\varphi_{i \lambda}(\tau, \hat{x})=\exp (\lambda \tau) g_{i j}(\tau, \hat{x}) f_{\lambda}^{j}(\hat{x}) \\
-\int_{0}^{\tau} d y \exp \left[\lambda\left(\tau-\frac{1}{2} y\right)\right] g_{i j}(\tau, \hat{x}) g^{j k}(y, \hat{x}) \hat{\nabla}_{k} f_{0_{\lambda}}(\hat{x}) .
\end{gathered}
$$

Now imposing Dirichlet boundary conditions one finds $f_{0 \lambda}=f_{\lambda}^{i}=0$, and hence $\varphi_{\lambda}=0$ for any $\lambda$. Thus, ghost fields do not contribute at all to the transition amplitudes. Note that this is a peculiar property of Barvinsky boundary conditions. The use of the axial gaugeaveraging functional does not imply, by itself, that the ghost should vanish identically, unless the whole ghost 1-form is set to zero at the boundary, as in our case.

As in the previous sections, we impose the boundary conditions (3.2) on the spatial components of metric perturbations. The other components of the field $h_{a b}$ vanish everywhere in the axial gauge and, of course, at the boundary. This means, by the way, that all components of metric perturbations vanish at the boundary. Hence all possible surface terms in the action vanish in this gauge, and any second-order differential operator is in fact symmetric in this particular case. 


\section{Boundary operators in Euclidean quantum gravity}

\section{1-loop divergence in the axial gauge}

In the absence of boundaries, there is indeed a rich literature on the axial gauge in quantum gravity and for quantized gauge theories [18-23]. In [18], the starting point was the analysis of infrared properties of quantum gravity in the axial gauge. It was then shown that gravitons decouple from the Faddeev-Popov ghosts, and that the leading infrared divergences exponentiate and vanish in the exponent in the scattering of gravitons for pure Einstein gravity. This led to a series of difficult 1-loop calculations, showing that the graviton self-energy is non-transverse and $n_{a}$-dependent $[19,20]$. In [21], all counterterms of quantum gravity were evaluated at 1-loop order in the axial gauge, whilst further progress for gauge theories was made in [22], and a comprehensive review appears in [23].

In this section, however, we are interested in the ultraviolet divergences of pure gravity in the presence of boundaries in the axial gauge. The framework under consideration is relevant for 1-loop quantum cosmology [17] and the 1-loop analysis of partition functions in Euclidean quantum gravity. Thus, unlike [19, 20], we do not study the graviton selfenergy, but we focus on the scaling properties of 1-loop quantum gravity encoded in the $\zeta(0)$ value [17]. The consideration of the axial gauge is suggested by the general scheme of section 2 for diffeomorphism-invariant boundary conditions, since all metric perturbations are then set to zero at the boundary in the axial gauge.

We begin our analysis by fixing the axial gauge by the Dirac delta in the path integral, i.e. without gauge averaging. Thus, metric perturbations satisfy the relation $h_{a b}=\Pi_{a b}^{c d} h_{c d}$ with $\Pi$ defined in (3.3). Hence the graviton operator $\Delta_{A}$ in the axial gauge is obtained by the projection of the operator in the quadratic part of the action 
Boundary operators in Euclidean quantum gravity

without the gauge-averaging term, i.e. $S_{2}=\int_{M} d^{4} x \sqrt{g} \frac{1}{2} h_{a b} \Delta^{a b, c d} h_{c d}$, as $\Delta_{A}=\Pi \Delta \Pi$. In flat Euclidean space the operator $\Delta$ reduces to the well known form $[24,25]$

$$
\begin{aligned}
\Delta^{a b, c d} & =-\left(g^{a(c} g^{d) b}-g^{a b} g^{c d}\right) \square-g^{c d} \nabla^{(a} \nabla^{b)}-g^{a b} \nabla^{(c} \nabla^{d)} \\
& +2 \nabla^{(a} g^{b)(c} \nabla^{d)}
\end{aligned}
$$

One should stress that the graviton operator $\Delta_{A}$ in the axial gauge depends, of course, on the vector $n^{a}$ through the projection operator $\Pi$. Since in the axial gauge $h=\Pi h$, the spectrum of the operator $\Delta_{A}$ can be obtained by studying the spectrum of the operator $\Delta$ in $(7.1)$

$$
\Delta_{a b}^{c d} h_{(\lambda) c d}=\lambda h_{(\lambda) a b}
$$

with the boundary conditions (3.2), or explicitly,

$$
\begin{aligned}
& -\square h_{(\lambda) a b}+g_{a b} \square h_{(\lambda)}-\nabla_{a} \nabla_{b} h_{(\lambda)}-g_{a b} \nabla_{c} \nabla_{d} h_{(\lambda)}^{c d}+2 \nabla_{c} \nabla_{(a} h_{(\lambda) b)}^{c} \\
& =\lambda h_{(\lambda) a b} .
\end{aligned}
$$

If one acts with the covariant differentiation operator on (7.3) one finds the equation

$$
\lambda \nabla^{a} h_{(\lambda) a b}=0
$$

which implies that, for any $\lambda \neq 0$, metric perturbations are transverse in flat 4-space. Moreover, the insertion of (7.4) into (7.3), jointly with multiplication by $g^{a b}$ and summation over repeated indices leads to

$$
\left(-\square+\frac{1}{2} \lambda\right) h_{(\lambda)}=0
$$




\section{Boundary operators in Euclidean quantum gravity}

It is indeed well known that the spectrum of the Laplace operator on compact manifolds is bounded from below [26]. Thus, for $\lambda$ greater than a positive constant, the operator

$-\square+\frac{1}{2} \lambda$ is positive-definite, and hence (7.5) implies that metric perturbations are traceless as well, i.e. $h=0$.

The only technical problems might arise with zero-modes, i.e. non-trivial eigenfunctions belonging to vanishing eigenvalues and satisfying the given boundary conditions. Although we are not (yet) able to prove a general theorem, we can however point out that, in the particular (and relevant) case of flat Euclidean 4-space bounded by a 3 -sphere, no non-trivial basis functions exist. This can be proved by inspection of the mode-bymode form of the coupled eigenvalue equations (2.5)-(2.11) of [27], jointly with equations (2.12) therein, which define the various operators acting on perturbative modes of the gravitational field.

Thus, since the ghost field vanishes identically in the axial gauge, as well as the normal components of $h_{a b}$, whilst $h_{i j}$ is only transverse-traceless and no non-trivial zero-modes exist, the resulting $\zeta(0)$ value coincides with the one first obtained in [28]

$$
\zeta(0)=\zeta_{\mathrm{TT}}(0)=-\frac{278}{45} .
$$

It is now instructive to outline the calculation when the gauge-averaging method is instead used. The axial-gauge functional modifies the operator (7.1) by the addition of the term $\frac{1}{\alpha} n^{(a} g^{b)(c} n^{d)}$. Thus, covariant differentiation of (7.3), and its contraction with $g^{a b}$, lead instead to the equations

$$
\frac{1}{2 \alpha}\left[\left(K_{b}^{c} n^{d}+K^{c d} n_{b}\right) h_{(\lambda) c d}+n_{b} n^{d} \nabla^{a} h_{(\lambda) a d}\right]
$$


Boundary operators in Euclidean quantum gravity

$$
\begin{aligned}
& +\frac{1}{2 \alpha}\left[(\operatorname{Tr} K) n^{d} h_{(\lambda) b d}+n^{d} \nabla_{(n)} h_{(\lambda) b d}\right] \\
& =\lambda \nabla^{a} h_{(\lambda) a b} \\
& \quad\left(-\square+\frac{1}{2} \lambda\right) h_{(\lambda)}=\frac{1}{2 \alpha} n^{c} n^{d} h_{(\lambda) c d}
\end{aligned}
$$

subject to the boundary conditions according to which the whole set of metric perturbations vanishes at the boundary. Indeed, in the particular case of flat Euclidean 4-space bounded by a 3 -sphere of radius $a$, the unperturbed extrinsic-curvature tensor $K_{i j}$ is equal to $\frac{1}{a} g_{i j}$, and $\nabla_{(n)} h_{(\lambda) b 0}$ vanishes $\forall b$ on choosing $n^{a}=(1,0,0,0)$, if $h_{00}=h_{0 i}=0$. Thus, a solution of (7.7) and (7.8) with the boundary conditions described above is compatible with having $h_{00}=h_{0 i}=0$ everywhere, whilst $h_{i j}$ is transverse-traceless (and hence $h_{a b}$ as

well). Moreover, this is the solution, since a unique, smooth and analytic solution exists of the quantum boundary-value problem for $h_{a b}$ with homogeneous Dirichlet conditions at the boundary.

\section{Concluding remarks}

Although the choice of boundary conditions is by no means unique in physics, the request of mathematical consistency may lead to severe restrictions, and this is indeed the case in Euclidean quantum gravity. Motivated by 1-loop quantum cosmology [17], this paper has studied the mathematical foundations of the boundary conditions for semiclassical gravity. The four basic properties one would like to respect are as follows:

(i) Invariance of the whole set of boundary conditions under infinitesimal diffeomorphisms on metric perturbations (see (2.1)). 


\section{Boundary operators in Euclidean quantum gravity}

(ii) Preservation of the boundary [29].

(iii) Local nature of the boundary operators. These should involve zero- and first-order differential operators, which may or may not represent (complementary) projectors.

(iv) Symmetry, and possibly essential self-adjointness, of the differential operators acting on metric perturbations and ghost 1-form.

Among the four different schemes studied so far in the literature [1, 8-10, 16, 30], attention has been focused in our paper on Barvinsky boundary conditions [8-10]. These are the only ones which require that the gauge-averaging functional $\Phi_{a}$ should vanish at the boundary. They provide a framework which is gauge-invariant by construction, and are local in that the boundary operators involve first-order or zero-order differential operators (cf [30]). The first result of our analysis is that, in the de Donder gauge, which leads to a minimal operator on metric perturbations, the boundary operators involve complementary projectors but also a nilpotent operator. This is a substantial difference with respect to the scheme proposed in [1], where only projection operators occur in the boundary conditions. In Euclidean quantum gravity, the resulting operator on metric perturbations is symmetric. Such a proof was lacking in the literature (cf [8] and [30]).

We have also shown that the boundary conditions (2.11a) and (2.12a) are compatible with the request (iv) also in non-covariant gauges. For example, on choosing the axialgauge functional, we have found that symmetry of the differential operators is immediately obtained (section 6). Moreover, the resulting 1-loop divergence has been found to coincide with the one resulting from transverse-traceless modes only [28]. This is a non-trivial property, since a gauge has been found such that the contributions of ghost and gauge 


\section{Boundary operators in Euclidean quantum gravity}

modes vanish separately in the presence of boundaries. This property is not shared by other non-covariant gauges, e.g. the Coulomb gauge for Euclidean Maxwell theory [31], where the ghost and gauge contributions cannot be made to vanish separately for problems with boundary. Note however that non-covariant choices, like the axial gauge $n^{b} h_{a b}=0$, might restrict the class of background four-geometries to those for which the singularity at the origin is avoided (e.g. the so-called two-boundary problem [7]), so that normal components of metric perturbations are well defined.

Last, we have put on solid ground the proof of symmetry of the graviton operator when the boundary conditions studied in [16] are imposed. It now remains to be seen whether such an operator is essentially self-adjoint (cf [32]), and whether the semiclassical theory is consistent despite the lack of complete gauge invariance of the boundary conditions (cf $[1,29,30])$. The former task appears easier, since one deals with a Laplace-like operator (in flat space) with Dirichlet and Robin sectors.

At a technical level, the outstanding open problem is now to find a geometric theory of heat-kernel asymptotics corresponding to the form (4.11) and (4.12) of Barvinsky boundary conditions in the de Donder gauge. The scheme is (far) more involved than the one considered in [2-4], since both normal and tangential derivatives of metric perturbations occur in the boundary conditions. However, such a step should be undertaken to complete the geometric description of ultraviolet divergences at 1-loop on manifolds with boundary.

Last, but not least, one has to prove essential self-adjointness [32] of the operator acting on metric perturbations when Barvinsky boundary conditions [10] in linear covariant gauges are imposed in the case of flat or curved four-dimensional backgrounds. 
Boundary operators in Euclidean quantum gravity

\section{Acknowledgments}

Anonymous referees made comments which led to a substantial improvement of the original manuscript, and we are indebted to Hugh Osborn for sending us a copy of [14]. The work of I Avramidi was supported by the Alexander von Humboldt Foundation, by the Istituto Nazionale di Fisica Nucleare and by the Deutsche Forschungsgemeinschaft. He is also grateful to the Naples Section of the INFN for hospitality. The work of A Kamenshchik

was partially supported by the Russian Foundation for Fundamental Researches through grant No 96-02-16220-a, and by the Russian Research Project "Cosmomicrophysics".

\section{References}

[1] Luckock H C 1991 J. Math. Phys. 321755

[2] Moss I G and Poletti S J 1994 Phys. Lett. 333B 326

[3] Vassilevich D V 1995 J. Math. Phys. 363174

[4] Gilkey P B 1995 Invariance Theory, the Heat Equation and the Atiyah-Singer Index Theorem (Boca Raton: CRC Press)

[5] D'Eath P D and Esposito G 1991 Phys. Rev. D 433234

[6] Kamenshchik A Yu and Mishakov I V 1993 Phys. Rev. D 471380

[7] Esposito G, Kamenshchik A Yu, Mishakov I V and Pollifrone G 1994 Class. Quantum Grav. 112939

[8] Esposito G, Kamenshchik A Yu, Mishakov I V and Pollifrone G 1995 Phys. Rev. D 523457 
Boundary operators in Euclidean quantum gravity

[9] Esposito G 1996 in Quantum Field Theory Under the Influence of External Conditions ed M Bordag (Stuttgart-Leipzig: Teubner)

[10] Barvinsky A O 1987 Phys. Lett. 195B 344

[11] Strocchi F 1993 Selected Topics on the General Properties of Quantum Field Theory (Singapore: World Scientific)

[12] Esposito G, Gionti G, Kamenshchik A Yu, Mishakov I V and Pollifrone G 1995 Int. J. Mod. Phys. D 4735

[13] Barvinsky A O, Kamenshchik A Yu and Karmazin I P 1992 Ann. Phys., NY 219201

[14] McAvity D M and Osborn H 1991 Class. Quantum Grav. 81445

[15] York J W 1986 Found. Phys. 16249

[16] Esposito G and Kamenshchik A Yu 1995 Class. Quantum Grav. 122715

[17] Esposito G 1994 Quantum Gravity, Quantum Cosmology and Lorentzian Geometries (Lecture Notes in Physics m12) (Berlin: Springer)

[18] Matsuki T 1979 Phys. Rev. D 192879

[19] Capper D M and Leibbrandt G 1982 Phys. Rev. D 251009

[20] Capper D M and Leibbrandt G 1982 Phys. Rev. D 252211

[21] Matsuki T 1985 Phys. Rev. D 323164

[22] Kreuzer M, Piquet O, Rebhan A and Schweda M 1986 Phys. Lett. 169B 221

[23] Leibbrandt G 1987 Rev. Mod. Phys. 591067

[24] Fierz M and Pauli W 1939 Proc. R. Soc. London A173 211

[25] Avramidi I G 1991 Int. J. Mod. Phys. A 61693

[26] Chavel I 1984 Eigenvalues in Riemannian Geometry (New York: Academic Press) 
Boundary operators in Euclidean quantum gravity

[27] Esposito G, Kamenshchik A Yu, Mishakov I V and Pollifrone G 1994 Phys. Rev. D $\mathbf{5 0} 6329$

[28] Schleich K 1985 Phys. Rev. D 321889

[29] Luckock H C and Moss I G 1989 Class. Quantum Grav. 61993

[30] Marachevsky V N and Vassilevich D V 1996 Class. Quantum Grav. 13645

[31] Esposito G and Kamenshchik A Yu 1994 Phys. Lett. 336B 324

[32] Esposito G, Morales-Técotl H A and Pimentel L O 1996 Class. Quantum Grav. 13 957 